\title{
The Receiving Antenna as a Linear Differential Operator: Application to Spherical Near-Field Scanning
}

\author{
ARTHUR D. YAGHJIAN, SENIOR MEMBER, IEEE, AND RONALD C. WITTMANN
}

\begin{abstract}
The general receiving antenna is represented as a linear differential operator converting the incident field and its spatial derivatives at a single point in space to an output voltage. The differential operator is specified explicitly in terms of the multipole coefficients of the antenna's complex receiving pattern. When the linear operator representation is applied to the special probes used in spherical near-field measurements, a probe-corrected spherical transmission formula is revealed that retains the form, applicability, and simplicity of the nonprobe-corrected equations. The new spherical transmission formula is shown to be consistent with the previous transmission formula derived from the rotational and translational addition theorems for spherical waves.
\end{abstract}

\section{INTRODUCTION}

$\mathrm{T}$ RADITIONALLY, the output voltage of a receiving antenna is expressed as an integral involving the electromagnetic field incident upon the antenna and the fields or currents produced by the antenna when it is radiating into free space [1]-[5]. In this paper, however, we express the output of a general receiving antenna in terms of derivatives of the incident field at a single arbitrarily chosen point in space to which the complex receiving pattern of the antenna is referenced. The coefficients of the derivatives are determined simply from the spherical mode coefficients of the receiving pattern of the antenna. The usefulness of this differential operator representation for an arbitrary receiving antenna becomes apparent in deriving directly a simple probe-corrected transmission formula for spherical near-field scanning. In particular, the differential operator representation for the scanning probe allows both a derivation and formulation of spherical near-field probe correction that is free of the rotational and translational addition theorems for spherical waves.

The derivation of the linear differential operator representation of a general receiving antenna begins in Section II-A with the Kerns plane-wave transmission integral [4], [5]. This plane-wave transmission integral expresses the output of the receiving antenna as a Fourier transform of the dot product of the receiving spectrum of the antenna and the radiating spectrum of the incident field. Since the receiving spectrum of the antenna is a far-field function, it is expanded in Section II$B$ in a series of vector spherical harmonics (multipole fields).

Manuscript received November 26, 1984; revised April 19, 1985.

A. D. Yaghjian is with the Electromagnetic Sciences Division, Rome Air Development Center, Hanscom AFB, MA 01731.

R. C. Wittmann is with the Electromagnetic Fields Division, National Bureau of Standards, Boulder, CO 80303.
When this spherical multipole expansion for the receiving spectrum of the probe is inserted into the plane-wave transmission integral, the resulting multipole operator can be transferred outside the integral to reveal in Section II-C the desired linear differential operator representation for the receiving antenna.

Physically, one can understand the differential operator representation by viewing the complex receiving pattern of an arbitrary antenna as the superposition of the receiving patterns of elementary dipoles. Consider an antenna with the receiving pattern of an electric dipole located at the point $0^{\prime}$. Such an antenna would measure the incident electric field at $0^{\prime}$ in the direction of the dipole [4, sec. III-6.1]. Similarly, an antenna with the receiving pattern of an elementary magnetic dipole would measure the incident magnetic field in the direction of the dipole. Now the complex receiving pattern of an arbitrary antenna can be expressed as the sum not only of electric and magnetic dipoles but also of higher order multipoles. Moreover, these higher order multipoles can be constructed from elementary dipoles superimposed by a progressively higher order limiting procedure at the chosen reference point $0^{\prime}[6$, sec. 3.12]. For example, quadrupoles would respond to the incident field and its first spatial derivatives, octupoles to the field and its first and second derivatives, and so on. In deriving the differential operator representation, Section II develops a systematic approach for determining the coefficients of the incident fields and their spatial derivatives that excite an arbitrary receiving antenna, in terms of the spherical multipole coefficients of the antenna's complex receiving pattern.

In Section III we apply the linear differential operator representation of the general receiving antenna to the special symmetric probes [8]-[10] used for taking near-field antenna measurements on a sphere surrounding a test antenna. For these special probes the general differential operator reduces immediately to one involving only the electric and magnetic fields of the test antenna and the radial derivatives of these fields. Expanding the electric and magnetic fields of the test antenna in a series of vector spherical waves, and applying the radial differential operator for the probe to the spherical expansion, results in a simple spherical transmission formula that can be solved directly for the spherical mode coefficients of the test antenna using the orthogonality of the vector spherical wave functions. In other words, the differential operator representation reveals that the special probes used in spherical scanning may be viewed as ideal dipole probes which 
measure an effective incident field, and which allow the modal coefficients of the test antenna to be computed simply from conventional orthogonality relations.

Finally, in Section IV the new probe-corrected spherical transmission formula is compared with the spherical transmission formula derived by Jensen and Wacker using rotational and translational addition theorems [7]-[10]. The JensenWacker transmission equation is first converted to the simplified form of the new transmission equation. Then plane-wave coupling integrals for the spherical modes are evaluated to show that the translated probe coefficients of the JensenWacker formulation agree with those of the new formulation.

Many of the results contained in this paper were briefly described in the previous publications [11], [12].

\section{Representation of the Receiving Antenna as a Linear DIFFERENTIAL OPERATOR}

\section{A. The Plane-Wave Transmission Integral}

A linear, but otherwise arbitrary receiving antenna in free space is illuminated by an incident electromagnetic field produced by an arbitrary $\mathrm{CW}$ source located to the left of the receiving antenna; see Fig. 1. The response of the receiving antenna, which we will refer to simply as the probe, can be expressed as a two-dimensional Fourier transform of the dot product of the plane-wave receiving spectrum $\bar{s}(\bar{K})$ of the probe and the radiating spectrum $\bar{b}(\bar{K})$ of the source [4],[5]. Specifically, with $\exp (-i \omega t)$ time dependence understood,

$$
b_{p}(\bar{r})=F \int_{-\infty}^{\infty} \int_{-\infty}^{\infty} \bar{s}(\bar{K}) \cdot \bar{b}(\bar{K}) e^{i \bar{K} \cdot \bar{r}} d \bar{K} .
$$

Kerns [4] refers appropriately to (1) as the plane-wave transmission integral. The output response $b_{p}$ of the probe is the coefficient of the propagating mode (at the chosen reference surface $S_{0}$ ) emerging from the probe's feed transmission line or waveguide. ${ }^{1}$ The output of the probe depends on the separation $\bar{r}$ between the chosen origins 0 and $0^{\prime}$ fixed in the radiating source and the receiving antenna, respectively. The plane-wave transmission integral (1) assumes that the probe translates without rotation with respect to the source, and that multiple reflections between the source and probe are negligible (or at least unchanged) when the probe translates. Equation (1) also assumes that the probe remains on the $\hat{z}$ side (the right side in Fig. 1) of the source. In other words, the probe and source region do not encroach upon each other with respect to the $z$-direction.

The mismatch factor $F$ is given by

$$
F=\left(1-\Gamma_{0} \Gamma_{L}\right)^{-1}
$$

where $\Gamma_{0}$ and $\Gamma_{L}$ are the reflection coefficients of the probe and its terminating load, respectively, at the reference surface $S_{0}$ in the probe feed (see Fig. 1).

\footnotetext{
${ }^{1}$ We are assuming a single mode of propagation in the antenna feed; multimode propagation can be treated similarly by expressing the response of each mode as a separate plane-wave transmission integral.
}

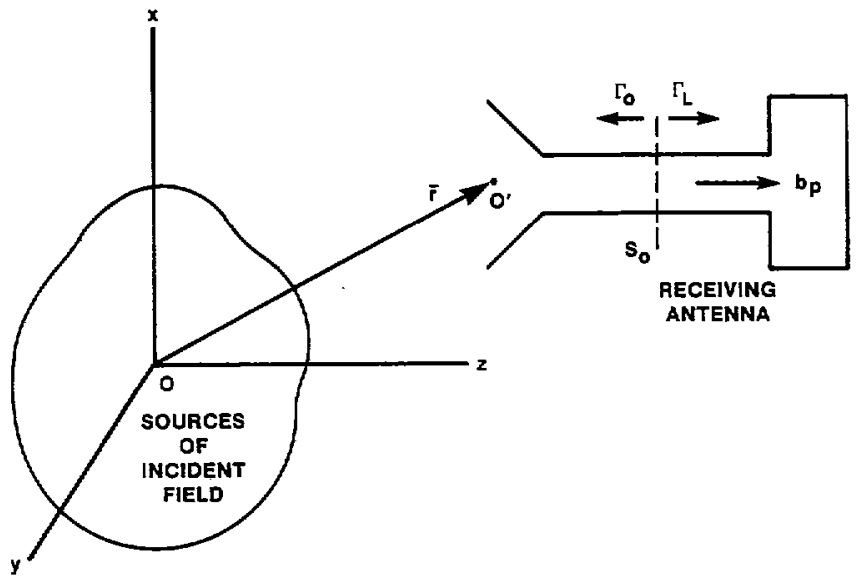

Fig. 1. Arbitrary linear receiving antenna (probe) in a $\mathrm{CW}$ incident field produced by sources to the left $(-\hat{z}$ side) of the probe.

The complete vectorial spectrum $\bar{b}(\bar{K})$ determines the electric and magnetic fields, $\widetilde{E}(\bar{r})$ and $\bar{H}(\bar{r})$, radiated by the source to the right of the source region in Fig. 1

$$
\begin{gathered}
\bar{E}(\bar{r})=\frac{1}{2 \pi} \int_{-\infty}^{\infty} \int_{-\infty}^{\infty} \bar{b}(\bar{K}) e^{i \bar{k} \cdot \bar{r}} d \bar{K} \\
\bar{H}(\bar{r})=\frac{1}{2 \pi Z_{o}} \int_{-\infty}^{\infty} \int_{-\infty}^{\infty} \hat{k} \times \bar{b}(\bar{K}) e^{i \bar{k} \cdot \bar{r}} d \bar{K} .
\end{gathered}
$$

The constant $Z_{0}$ is the impedance of free space and the caret designates unit vectors. The International System of Units (mksA) is used throughout.

An asymptotic evaluation of ( $3 a$ ) shows that the radiating spectrum $\bar{b}(\bar{K})$ is proportional to the far-field function of the source [4]; specifically

$$
-i \gamma \bar{b}(\bar{K})=\bar{E}(\hat{r}=\hat{k}) r e_{r \rightarrow \infty}^{-i k r} \equiv \bar{f}(\bar{K}) .
$$

Reciprocity implies that the receiving spectrum $\bar{s}(\bar{K})$ (i.e., the complex vectorial receiving pattern as a function of $\bar{K}$ ) of the probe is proportional to the far electric field of the probe when the probe is transmitting [4], [5]. (For nonreciprocal probes the receiving spectrum is proportional to the far electric field of the "adjoint" probe [4].) Thus, both $\bar{b}(\bar{K})$ and $\bar{s}(\bar{K})$ are farfield functions with no radial components, i.e.,

$$
\begin{aligned}
& \vec{b}(\bar{K}) \cdot \bar{k}=0 \\
& \bar{s}(\bar{K}) \cdot \bar{k}=0 .
\end{aligned}
$$

The phase references for the radiating and receiving spectra are the chosen origins 0 and $0^{\prime}$, respectively.

The double integration in (1) spans the transverse part $\bar{K}$ of the propagation vector $\bar{k}$, where

$$
\begin{gathered}
\bar{k}=\bar{K}+\gamma \hat{z} \\
\bar{K}=k_{x} \hat{x}+k_{y} \hat{y} \\
d \bar{K}=d k_{x} d k_{y}
\end{gathered}
$$


and

$$
\gamma=\left(k^{2}-K^{2}\right)^{1 / 2}
$$

is positive real or imaginary (assuming the frequency $\omega$ is greater than zero). The magnitude of $\bar{k}$ is simply $k=\omega / c, c$ being the speed of light in free space, and the magnitude of $\bar{K}$ is denoted by $K$. The integration covers both the propagating $(K<k)$ and evanescent $(K>k)$ parts of the spectra.

\section{B. A Multipole Expansion for the Receiving Spectrum of the Probe}

Because the receiving spectrum $\bar{s}(\bar{K})$ is proportional to the far electric field of the probe (adjoint probe, if the probe is nonreciprocal), it can be expanded in a series of vector spherical harmonics [6], [13]. Specifically,

$$
\bar{S}(\bar{K})=\sum_{l=1}^{\infty} \sum_{m=-l}^{l}\left[C_{l m}^{E} \bar{N}_{l m}(\bar{K})+C_{l m}^{H} \bar{M}_{l m}(\bar{K})\right]
$$

where $\left(C_{l m}^{E}, C_{l m}^{H}\right)$ are the electric and magnetic spherical mode (multipole) coefficients, and $\left(\bar{N}_{l m}, \bar{M}_{l m}\right)$ are the angularly dependent vector spherical harmonics (multipole far fields),

$$
\begin{aligned}
& \bar{M}_{l m}(\bar{K})=\bar{N}_{l m}(\bar{K}) \times \hat{K}, \quad \bar{N}_{l m}(\bar{K})=\hat{K} \times \bar{M}_{l m}(\bar{K}) \\
& \bar{N}_{l m}(\bar{K})=k \nabla\left[P_{l}^{m}\left(\frac{\gamma}{k}\right)\left(\frac{k_{x}}{K}+i \frac{k_{y}}{K}\right)^{m}\right] A_{l m}
\end{aligned}
$$

The $P_{l}^{m}$ are the associated Legendre functions as defined by Stratton [6]. The $A_{l m}$ are arbitrary real normalization factors included in (7) to facilitate comparisons with the various definitions of $\left(\bar{N}_{l m}, \bar{M}_{l m}\right)$ used by different authors. The gradient in (7b) differentiates with respect to the $\bar{k}$ coordinates. Equations (7) can be put into their more familiar form involving the spherical angles $\theta$ and $\phi$ if one associates $\vec{k}$ with $\bar{r}, \gamma / k$ with $\cos \theta, \tan ^{-1} k_{y} / k_{x}$ with $\phi$, and then notes that $\left(k_{x} /\right.$

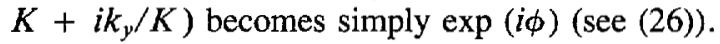

We want to substitute the receiving spectrum (6) into (1) and express the integrand so that (1) can be rewritten in terms of the source electric and magnetic fields (and their spatial derivatives) incident upon the probe. We know that if the probe were an ideal electric or magnetic dipole (i.e., if only $\bar{N}_{1 m}$ or $\bar{M}_{1 m}$ for $m=0$ and \pm 1 had nonzero coefficients in (6)), then the output $b_{p}$ of the probe in (1) would be proportional to the incident electric or magnetic field, respectively, at the reference point $0^{\prime}$ of the probe $[4, \mathrm{sec}$. III-6.1]. Thus, if one could express all the multipole fields in terms of $\bar{N}_{1 m}$ and $\bar{M}_{1 m}$ with $m=0$ and \pm 1 only, one may be able to find the desired linear differential operator representation for the probe response. Indeed, substitution from [6] of the definitions of the associated Legendre functions $P_{l}^{m}$ and $d P_{l}^{m} /$ $d \eta$, i.e.,

$$
P_{l}^{m}(\eta)=\left(1-\eta^{2}\right)^{m / 2} \frac{d^{m} P_{l}(\eta)}{d \eta^{m}}, \quad m \geq 0
$$

$$
\frac{d P_{l}^{m}(\eta)}{d \eta}=\left(1-\eta^{2}\right)^{m / 2}\left(\frac{d^{m+1} P_{l}}{d \eta^{m+1}}-\frac{m \eta}{1-\eta^{2}} \frac{d^{m} P_{l}}{d \eta^{m}}\right)
$$

into the definitions (7) of $\bar{N}_{l m}$ and $\bar{M}_{l m}$ leads to the following expressions for the multipole fields in terms of the electric and magnetic dipole fields:

$$
\begin{gathered}
\bar{N}_{l m}(\bar{K})=(\alpha+i \beta)^{m-1}\left[F_{l m}(\eta) \bar{N}_{11}+i G_{l m}(\eta) \bar{M}_{11}\right] / A_{11} \\
\bar{M}_{l m}(\bar{K})=(\alpha+i \beta)^{m-1}\left[F_{l m}(\eta) \bar{M}_{11}-i G_{l m}(\eta) \bar{N}_{11}\right] / A_{11} \\
\bar{N}_{l 0}(\bar{K})=\bar{N}_{10} G_{l 10} / A_{10} \\
\bar{M}_{l 0}(\bar{K})=\bar{M}_{10} G_{l 0} / A_{10}
\end{gathered}
$$

The symbols $\alpha, \beta$, and $\eta$ stand for $k_{x} / k, k_{y} / k$, and $\gamma / k$, respectively. The functions $F_{l m}$ and $G_{l m}$ are finite-order polynomials in $\eta$ that are conveniently expressed in terms of derivatives of Legendre polynomials:

$$
\begin{gathered}
F_{l m}(\eta)=\left[\eta \frac{d^{m+1} P_{l}(\eta)}{d \eta^{m+1}}+m \frac{d^{m} P_{l}(\eta)}{d \eta^{m}}\right] A_{l m} \\
G_{l m}(\eta)=\frac{d^{m+1} P_{l}(\eta)}{d \eta^{m+1}} A_{l m} .
\end{gathered}
$$

The modes $\bar{N}_{11}$ and $\bar{N}_{10}$ represent the far electric fields of electric dipoles oriented in the transverse $(x, y)$ and longitudinal $(z)$ directions, respectively. Similarly, $\bar{M}_{11}$ and $\bar{M}_{10}$ are the far electric fields of magnetic dipoles oriented in the transverse and longitudinal directions. They can be written explicitly as

$$
\begin{gathered}
\bar{N}_{11} / A_{11}=(\hat{x}+i \hat{y})-\hat{k}(\alpha+i \beta) \\
\bar{M}_{11} / A_{11}=(\hat{x}+i \hat{y}) \times \hat{k} \\
\bar{N}_{10} / A_{10}=\hat{z}-\eta \hat{k} \\
\bar{M}_{10} / A_{10}=\hat{z} \times \hat{k} .
\end{gathered}
$$

Equations (9a) and (9b) determine $\bar{N}_{l m}$ and $\bar{M}_{l m}$ for positive values of $m$. For negative integers $m$, the vector spherical harmonics can be defined conveniently by the simple relations

$$
\begin{aligned}
& \bar{N}_{l,-m}=(-1)^{m} \bar{N}_{l m}^{*} \\
& \bar{M}_{l,-m}=(-1)^{m} \bar{M}_{l m}^{*}
\end{aligned}
$$

where the asterisk denotes the complex conjugate.

\section{Transformation of the Plane-Wave Transmission Integral by the Multipole Expansion of the Probe's Receiving Spectrum}

When the dipolar expressions (9) and (12) for the multipole fields are inserted into (6), the receiving spectrum $\bar{s}(\bar{K})$ takes the form of polynomials in $\alpha, \beta$, and $\eta$ multiplied by $\bar{N}_{11}, \bar{M}_{11}$, 


$$
\begin{aligned}
& \bar{N}_{10} \text { and } \bar{M}_{10}: \\
& \bar{s}(\bar{K})=\frac{\bar{N}_{11}}{A_{11}} \sum_{l=1}^{\infty} \sum_{m=1}^{l}\left[C_{l m}^{E} F_{l m}(\eta)-i C_{l m}^{H} G_{l m}(\eta)\right](\alpha+i \beta)^{m-1} \\
& +\frac{\bar{M}_{11}}{A_{11}} \sum_{l=1}^{\infty} \sum_{m=1}^{l}\left[C_{l m}^{H} F_{l m}+i C_{l m}^{E} G_{l m}\right](\alpha+i \beta)^{m-1} \\
& +\frac{\bar{N}_{l 1}^{*}}{A_{11}} \sum_{l=1}^{\infty} \sum_{m=1}^{l}(-1)^{m}\left[C_{l,-m}^{E} F_{l m}+i C_{l,-m}^{H} G_{l m}\right](\alpha-i \beta)^{m-1} \\
& +\frac{\bar{M}_{11}^{*}}{A_{11}} \sum_{l=1}^{\infty} \sum_{m=1}^{l}(-1)^{m}\left[C_{l,-m}^{H} F_{l m}-i C_{l,-m}^{E} G_{l m}\right](\alpha-i \beta)^{m-1} \\
& +\frac{1}{A_{10}} \sum_{l=1}^{\infty}\left[C_{l 0}^{E} \bar{N}_{10}+C_{l 0}^{H} \bar{M}_{10}\right] G_{l 0} .
\end{aligned}
$$

With the help of the explicit expressions (11) for $\bar{N}_{11}, \bar{M}_{11}, \bar{N}_{10}$ and $\bar{M}_{10},(13)$ can be written as follows in terms of the $\hat{x}, \hat{y}, \hat{z}$ unit vectors:

$$
\begin{aligned}
\bar{s}(\bar{K})= & \hat{x} \mathscr{L}_{x}^{E}(\alpha, \beta, \eta)+\hat{y} \mathcal{L}_{y}^{E}(\alpha, \beta, \eta)+\hat{z} \mathscr{L}_{z}^{E}(\alpha, \beta, \eta) \\
& +\hat{x} \times \hat{k} \mathscr{L}_{x}^{H}(\alpha, \beta, \eta) \\
& +\hat{y} \times \hat{k} \mathscr{L}_{y}^{H}(\alpha, \beta, \eta)+\hat{z} \times \hat{K} \mathscr{L}_{z}^{H}(\alpha, \beta, \eta)
\end{aligned}
$$

where the polynomials $\mathscr{L}$ are defined by

$$
\begin{aligned}
\mathfrak{L}_{\substack{y \\
y}}^{(E, H)}=\left(\begin{array}{c}
1 \\
i
\end{array}\right) \sum_{l=1}^{\infty} \sum_{m=1}^{l}\left\{F _ { l m } \left[C_{l m}^{(E, H)}(\alpha+i \beta)^{m-1}\right.\right. \\
\left. \pm C_{l,-m}^{(E, H)}(\alpha-i \beta)^{m-1}(-1)^{m}\right] \\
(-,+) i G_{l m}\left[C_{l m}^{(H, E)}(\alpha+i \beta)^{m-1}\right. \\
\left.\left.\mp C_{l,-m}^{(H, E)}(\alpha-i \beta)^{m-1}(-1)^{m}\right]\right\}
\end{aligned}
$$

$\mathcal{L}_{z}^{(E, H)}=\sum_{i=1}^{\infty} C_{l 0}^{(E, H)} G_{l 0}$

The $\bar{k}$ part of $\bar{N}_{11}$ in (11a) and $\bar{N}_{10}$ in (11c) has been dropped in (14a) because $\bar{k} \cdot \bar{b}(\bar{K})$ is zero and thus the $\bar{k}$ part of $\bar{N}_{11}$ and $\bar{N}_{10}$ will not contribute when $\bar{s}(\bar{K})$ is substituted into (1).

Consider the substitution of the multipole expansion (14) for the receiving spectrum $\bar{s}(\bar{K})$ into the transmission integral (1). Since the $\mathcal{L}$ in (14) are all polynomial functions of $\alpha, \beta$, and $\eta$, and $\bar{k}$ equals $k(\alpha \hat{x}+\beta \hat{y}+\eta \hat{z})$, the $\mathscr{L}$ can all be transferred to the left of the integral sign in (1) if $\alpha, \beta$, and $\eta$ are merely replaced by the partial derivatives,

$$
\alpha \rightarrow \frac{1}{i k} \frac{\partial}{\partial x}
$$

$$
\begin{gathered}
\beta \rightarrow \frac{1}{i k} \frac{\partial}{\partial y} \\
\eta \rightarrow \frac{1}{i k} \frac{\partial}{\partial z} .
\end{gathered}
$$

This operator transfer leaves only the functions $\bar{b}(\bar{K})$ and $\bar{k} \times$ $\bar{b}(\bar{K})$ multiplied by $\exp (i \vec{k} \cdot \vec{r})$ in the integrand of (1). Since these remaining integrals define the electric and magnetic fields (see (3a), (3b)), (1) reduces to

$$
\begin{aligned}
\frac{b_{p}(\bar{r})}{2 \pi F}=\left[\mathcal{L}_{x}^{E} E_{x}(\bar{r})\right. & \left.+\mathcal{L}_{y}^{E} E_{y}(\bar{r})+\mathcal{L}_{z}^{E} E_{z}(\bar{r})\right] \\
& +Z_{o}\left[\mathcal{L}_{x}^{H} H_{x}(\bar{r})+\mathcal{L}_{y}^{H} H_{y}(\bar{r})+\mathscr{L}_{z}^{H} H_{z}(\bar{r})\right]
\end{aligned}
$$

or in more compact vector notation

$$
\frac{b_{p}(\bar{r})}{2 \pi F}=\overline{\mathfrak{L}}_{E} \cdot \bar{E}(\bar{r})+Z_{o} \overline{\mathfrak{L}}_{H} \cdot \bar{H}(\bar{r})
$$

where $\overline{\mathfrak{L}}_{E}$ and $\overline{\mathcal{L}}_{H}$ are the linear differential operators formed by replacing $\alpha, \beta$, and $\eta$ in (14) with the $x, y$, and $z$ partial derivatives in (15).

Equations (16) represent the output of an arbitrary linear receiving antenna in terms of the incident electric and magnetic fields (incident from the $-\hat{z}$ side of the antenna) and their spatial derivatives at the point $0^{\prime}$ to which the receiving spectrum of the antenna is referenced. The linear differential operators $\overline{\mathfrak{L}}_{E}$ and $\overline{\mathfrak{L}}_{H}$ are defined by (14) directly in terms of the coefficients of the electric and magnetic multipole fields of the receiving spectrum of the probe, phase referenced to $0^{\prime}$. In other words, if one computes or measures the complex vectorial receiving pattern of an antenna, one can compute the spherical mode coefficients of that far-field function, insert those multipole coefficients into (14b) and (14c), replace $\alpha, \beta$, and $\eta$ by the derivatives in (15), and determine from (16) the response of the receiving antenna to an arbitrary incident field produced by sources on the $-\hat{z}$ side of the probe.

Although (16) requires both the incident electric and magnetic fields to determine the response of the receiving antenna, (16) could be rewritten in terms of the incident $\bar{E}$ or $\bar{H}$ alone merely be representing $\bar{E}$ or $\bar{H}$ as the curl of the other. However, we retain the form (16) because it shows only the azimuthally symmetric multipoles $(m=0)$ responding directly to the longitudinal $(z)$ components of the incident fields, and because it proves convenient for application to spherical near-field scanning.

If the probe in Fig. 1 is moved along the $+z$ axis to the far field of the source so that the probe is illuminated by the planewave field

$$
\begin{gathered}
\bar{E}(\bar{r})=\bar{E}_{t}^{0} e^{i k z} \\
\bar{H}(\bar{r})=\hat{z} \times \bar{E}_{t}^{0} / Z_{o} e^{i k z}
\end{gathered}
$$

equations (16) predict the familiar result that the output of the probe in a plane-wave field is proportional to the transverse electric or magnetic field $\left(\bar{E}_{t}^{0}\right.$ or $\left.\bar{H}_{t}^{0}\right)$. To see this, note that $E_{z}$ and $H_{z}$ are zero for an incident plane wave propagating along the $z$-axis, all the transverse $(x, y)$ 
derivatives in (16) are zero because the plane-wave incident field does not vary in the transverse direction, and the longitudinal derivatives $(\eta=\partial / i k \partial z)$ in (16) become unity because the plane wave has exp (ikz) dependence. Thus, $\overline{\mathscr{L}}_{E}$ and $\overline{\mathfrak{L}}_{H}$ reduce to constant vectors $\left(\bar{C}_{E}\right.$ and $\left.\bar{C}_{H}\right)$ and $(16 \mathrm{~b})$ becomes

$$
\begin{aligned}
\frac{b_{p}(\bar{r})}{2 \pi F}=\bar{C}_{E} \cdot \bar{E}_{t}^{0}+Z_{o} \bar{C}_{H} \cdot \bar{H}_{t}^{0} & =\left[\bar{C}_{E}+\bar{C}_{H} \times \hat{z}\right] \cdot \bar{E}_{t}^{0} \\
& =Z_{o}\left[\bar{C}_{H}-\bar{C}_{E} \times \hat{z}\right] \cdot \bar{H}_{t}^{0} .
\end{aligned}
$$

\section{Application to Spherical Near-Field Scanning}

In general, the far fields of antennas can be determined accurately from measurements in the near field only if one corrects for the response of the measurement probe [14]-[17]. For planar [4], [15], [18] and cylindrical [19]-[21] near-field scanning, the probe-corrected formulation is hardly more complicated than the uncorrected formulation that assumes the probe measures the electric or magnetic field at each point in space, i.e., the probe responds as an ideal electric or magnetic dipole. However, the formulation of probe correction for spherical scanning [7], and its practical realization using symmetric probes [8]-[10], [22], involve rotational and translational addition theorems that lead to coupling equations and deconvolution theorems that are considerably more complicated than those of planar, cylindrical, or nonprobecorrected spherical scanning. Thus, the primary aim of this section is to provide a new, yet rigorous formulation for probe-corrected spherical near-field measurements [11], [12] that retains the simplicity of the uncorrected formulation and thus the practicality of the probe-corrected planar and cylindrical near-field formulations. The new formulation proceeds naturally and directly from the differential operator representation for the probe that was derived in Section II.

\section{A. Differential Operator Representation for the Spherical Scanning Probe}

Consider a receiving probe scanning in $\phi$ and $\theta$ on an imaginary sphere of radius $r$ centered at a chosen origin 0 and enclosing a radiating test antenna, as shown in Fig. 2. (In practice, of course, the test antenna usually rotates and the probe stays fixed; for ease of visualization we assume the converse.) Unlike the probe in Fig. 1 which remained parallel to the $z$-axis, the spherical probe of Fig. 2 remains fixed in the radial axis as it scans the test antenna. Moreover, we assume that complete polarization data for the test antenna is required, and thus the probe scans in two spin orientations, $\psi=0$ and $\psi$ $=90^{\circ}$. A convenient vector response $\bar{b}_{t}$ for the probe can be defined as a combination of the scalar responses in these two orientations;

$$
\bar{b}_{t}=b_{p}\left(\psi=0^{\circ}\right) \hat{\theta}+b_{p}\left(\psi=90^{\circ}\right) \hat{\phi} .
$$

For example, if the probe in the $\psi=0^{\circ}$ orientation can be represented by an ideal electric or magnetic dipole parallel to $\hat{\theta}, \bar{b}_{t}$ is proportional to the incident electric or magnetic field transverse to the radial direction.

We can apply the differential operator representation (16) to

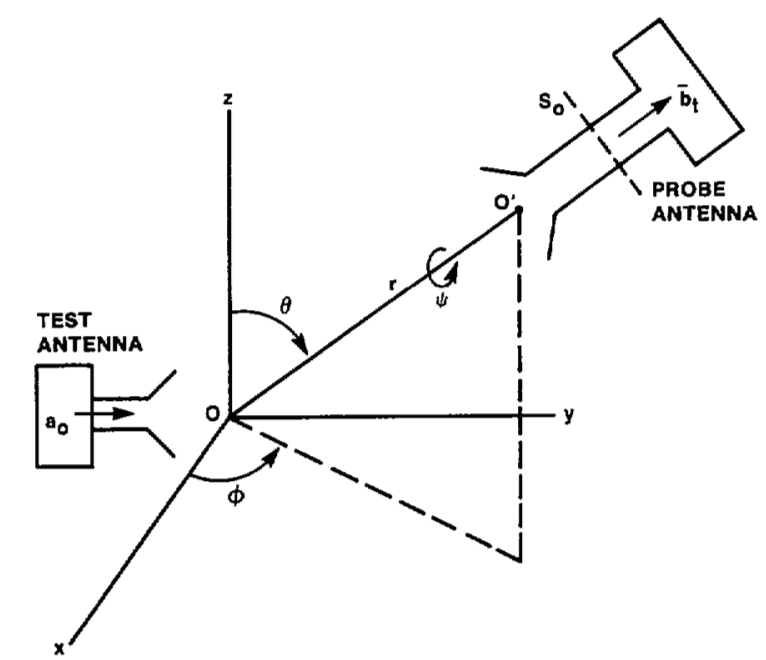

Fig. 2. Receiving probe scanning on an imaginary sphere enclosing the radiating test antenna; $\bar{b}_{t}=b_{p}\left(\psi=0^{\circ}\right) \hat{\hat{\theta}}+b_{p}\left(\psi=90^{\circ}\right) \hat{\dot{\phi}}$.

the spherical scanning probe of Fig. 2 if we choose the $z$ coordinate of (16) parallel to the radial direction. In particular, $\eta$ in (16) becomes $\partial / i k \partial r$ instead of $\partial / i k \partial z$. In addition, probes commonly used for spherical near-field scanning are constructed so that their receiving multipole coefficients satisfy the relations [8]-[10]:

$$
\begin{gathered}
C_{l m}^{E_{l} H}=0 \quad m \neq \pm 1 \\
C_{l 1}^{E}=C_{l,-1}^{E} \equiv C_{l}^{E} \\
C_{l \mathrm{I}}^{H}=-C_{l,-1}^{H} \equiv C_{l}^{H} .
\end{gathered}
$$

For these $m= \pm 1$ probes, which have the azimuthal symmery of $\hat{\phi}$-directed electric dipoles and $\hat{\theta}$-directed magnetic dipoles, the transverse derivatives in the operators of (16) vanish, leaving just the derivatives with respect to the longitudinal direction ( $r$ in Fig. 2).

Substitution of the relations (20) into (14b) shows that $\mathfrak{L}_{X}^{E}$, $\mathcal{L}_{y}^{H}$, and $\mathcal{L}_{z}^{E}$ for these probes are zero and that $\mathcal{L}_{y}^{E}$ and $\mathcal{L}_{x}^{H}$ reduce to

$$
\begin{aligned}
& L_{E}=\mathscr{L}_{y}^{E}=2 i \sum_{l=1}^{\infty}\left(C_{l}^{E} F_{l}-i C_{l}^{H} G_{l}\right) \\
& L_{H}=\mathscr{L}_{x}^{H}=2 \sum_{l=1}^{\infty}\left(C_{l}^{H} F_{l}+i C_{l}^{E} G_{l}\right) .
\end{aligned}
$$

The operators $F_{l}$ and $G_{l}$ in (21), which involve radial derivatives only, are found from the definitions (10) by setting $m=1$ and $\eta=\partial / i k \partial r$, to get

$$
\begin{gathered}
F_{l}=\frac{d}{d \eta}\left[\eta \frac{d P_{l}(\eta)}{d \eta}\right] \\
A_{l}\left(\eta=\frac{1}{i k} \frac{\partial}{\partial r}\right) \\
G_{l}=\frac{d^{2} P_{l}(\eta)}{d \eta^{2}} A_{l} .
\end{gathered}
$$

With (21) inserted into (16), and (16) into (19), the vector 
output response $\bar{b}_{t}$ of the probe becomes simply

$$
\frac{\bar{b}_{t}}{2 \pi F}=L_{E}\left[\bar{E}_{t} \times \hat{r}\right]+Z_{o} L_{H}\left[\bar{H}_{t}\right]
$$

where $\bar{E}_{t}(\bar{r})$ and $\bar{H}_{t}(\bar{r})$ are the transverse (with respect to $\bar{r}$ ) fields of the test antenna. Again, note that if the probe has the complex receiving pattern (far field) of an ideal electric dipole located at $0^{\prime}$, all the probe coefficients are zero except $C_{1}^{E}$; since $F_{1}=1$ and $G_{1}=0, L_{H}$ is zero, $L_{E}$ is equal to the constant $2 F i C_{1}^{E}$, and the vector output $\bar{b}_{t}$ of the probe becomes proportional to the incident transverse electric field (cross $\hat{f}$ ) at the point $0^{\prime}$. Similarly for a probe with the receiving pattern of an ideal magnetic dipole, all probe coefficients are zero except $C_{1}^{H}$, and $\bar{b}_{t}$ becomes proportional to the incident transverse magnetic field. This close relationship (23) of the vector output of the probe to the incident transverse electromagnetic fields permits a straightforward derivation of a probe-corrected spherical transmission formula [11] which is free of complicated rotational and translational addition theorems, and which approaches the simplicity of the nonprobe-corrected formulas involving only the transverse $E$ - or $H$-field.

\section{B. Derivation of Simplified Probe-Corrected Spherical Transmission Formula}

The fields outside the smallest sphere centered at the chosen origin 0 and circumscribing the test antenna which radiates into free space can be expanded in a series of vector spherical harmonics [6], [13]. In particular, the transverse electric and magnetic fields $\left(\bar{E}_{t}^{A}, \bar{H}_{t}^{A}\right)$ of the test antenna can be written in the form

$$
\begin{gathered}
\bar{E}_{t}^{A}(r, \phi, \theta)=\sum_{n=1}^{\infty} \sum_{m=-n}^{n}\left[b_{n m}^{E} g_{n}(k r) \bar{N}_{n m}+b_{n m}^{H} f_{n}(k r) \bar{M}_{n m}\right] \\
\bar{H}_{i}^{A}(r, \phi, \theta)=\frac{1}{i Z_{o}} \sum_{n=1}^{\infty} \sum_{m=-n}^{n} \\
\quad\left[b_{n m}^{H} g_{n}(k r) \bar{N}_{n m}+b_{n m}^{E} f_{n}(k r) \bar{M}_{n m}\right] .
\end{gathered}
$$

The constants $\left(b_{n m}^{E}, b_{n m}^{H}\right)$ are the electric and magnetic multipole coefficients of the test antenna, $\left(f_{n}, g_{n}\right)$ are the radially dependent spherical Hankel functions

$$
\begin{gathered}
f_{n}(x)=h_{n}^{(1)}(x) \\
g_{n}(x)=\frac{1}{x} \frac{d}{d x}\left(x h_{n}^{(1)}(x)\right)
\end{gathered}
$$

and $\left(\bar{N}_{n m}, \bar{M}_{n m}\right)$ are the angularly dependent vector spherical harmonics defined previously in (7) as functions of $\bar{K}$ but repeated here as functions of $\phi$ and $\theta$,

$$
\begin{gathered}
\bar{M}_{n m}=\bar{N}_{n m} \times \hat{r}, \bar{N}_{n m}=\hat{r} \times \bar{M}_{n m} \\
\bar{N}_{n m}(\phi, \theta)=r \nabla\left[P_{n}^{m}(\cos \theta) e^{i m \phi}\right] A_{n m} .
\end{gathered}
$$

The coefficient $a_{0}$ of the propagating mode that feeds the test antenna in Fig. 2 is absorbed into the multipole coefficients $\left(b_{n m}^{E}, b_{n m}^{H}\right)$.
If multiple reflections between the probe and test antenna are negligible, the fields (24) of the test antenna in free space can be substituted for the $\left(\bar{E}_{t}, \bar{H}_{t}\right)$ fields in the differential operator representation (23) for the spherical scanning probe. This substitution of (24) into (23) yields immediately a simple, yet rigorous probe-corrected spherical transmission formula in terms of the familiar vector spherical harmonics:

$$
\begin{aligned}
\frac{\bar{b}_{t}(r, \phi, \theta)}{2 \pi F}=\sum_{n=1}^{\infty} \sum_{m=-n}^{n} & {\left[b_{n m}^{H}\left(L_{H} \frac{g_{n}}{i}-L_{E} f_{n}\right) \bar{N}_{n m}\right.} \\
& \left.-i b_{n m}^{E}\left(L_{H} f_{n}-L_{E} \frac{g_{n}}{i}\right) \bar{M}_{n m}\right]
\end{aligned}
$$

The transmission formula (27) expands the vector response of the probe in the same orthogonal vector spherical harmonics that expand the electric and magnetic field (24) of the test antenna. The only difference between (27) and (24) is in the radial functions. In (24) the radial functions involve just the spherical Hankel functions $f_{n}$ and $g_{n}$. In (27) the radial functions involve, through $L_{E}$ and $L_{H}$, the multipole coefficients $\left(C^{H}, C^{H}\right)$ of the complex receiving pattern of the probe as well as $f_{n}$ and $g_{n}$.

The unknown spherical mode coefficients $\left(b_{n m}^{E}, b_{n m}^{H}\right)$ can be found from the measured output $\bar{b}_{t}(r, \phi, \theta)$ of the probe with the help of the following orthogonality relations for $\bar{N}_{n m}$ and $\bar{M}_{n m}$ :

$$
\begin{aligned}
\int_{0}^{2 \pi} \int_{0}^{\pi} \bar{N}_{n m}(\phi, \theta) \cdot \bar{N}_{n^{\prime} m^{\prime}}^{*}(\phi, \theta) \sin \theta d \theta & d \phi \\
& =\frac{\delta_{n n^{\prime}} \delta_{m m^{\prime}}}{\Delta_{n m}}
\end{aligned}
$$

$$
\begin{aligned}
\int_{0}^{2 \pi} \int_{0}^{\pi} \bar{M}_{n m}(\phi, \theta) \cdot \bar{M}_{n^{\prime} m^{\prime}}^{*}(\phi, \theta) \sin \theta d \theta d \phi \\
=\frac{\delta_{n n^{\prime}} \delta_{m m^{\prime}}}{\Delta_{n m}}
\end{aligned}
$$

$$
\int_{0}^{2 \pi} \int_{0}^{\pi} \bar{N}_{n m}(\phi, \theta) \cdot \bar{M}_{n^{\prime} m^{\prime}}^{*}(\phi, \theta) \sin \theta d \theta d \phi=0
$$

$$
\Delta_{n m}=\frac{(2 n+1)(n-m) !}{4 \pi A_{n m}^{2} n(n+1)(n+m) !} .
$$

Dotting $\bar{M}_{n m}^{*}$ and $\bar{N}_{n m}^{*}$ into (27), integrating with $\theta$ and $\phi$ over the measurement sphere, and applying the orthogonality relations (28), one obtains

$$
\begin{gathered}
b_{n m}^{E}=\frac{\Delta_{n m}}{2 \pi F i\left[L_{E} \frac{g_{n}}{i}-L_{H} f_{n}\right]} \int_{0}^{2 \pi} \int_{0}^{\pi} \bar{b}_{t}(r, \phi, \theta) \\
b_{n m}^{H}=\frac{\Delta_{n m}}{2 \pi F\left[L_{H} \frac{g_{n}}{i}-L_{E} f_{n}\right]} \int_{0}^{2 \pi} \int_{0}^{\pi} \bar{b}_{t}(r, \phi) \sin \theta d \theta d \phi \\
\cdot \bar{N}^{*}(\phi, \theta) \sin \theta d \theta d \phi .
\end{gathered}
$$


Once the modal coefficients of the test antenna are computed from (29), the fields outside the test antenna can be determined by substituting these constant coefficients back into the summations (24). In particular, the far fields of the test antenna, obtained by letting the radius $r$ approach infinity in (24a) or (24b), take the form

$$
\begin{aligned}
\bar{E}^{A}(r, \phi, \theta)_{r \rightarrow \infty}=\frac{e^{i k r}}{i k r} \sum_{n=1}^{\infty} \sum_{m=-n}^{n}(-i)^{n} \\
\cdot\left[i b_{n m}^{E} \bar{N}(\phi, \theta)+b_{n m}^{H} \bar{M}(\phi, \theta)\right] \\
\bar{H}^{A}(r, \phi, \theta)=\hat{r} \times \bar{E}^{A}(r, \phi, \theta) / Z_{o} .
\end{aligned}
$$

The orthogonality integrals in (29a) and (29b) required to determine the spherical mode coefficients are identical to those that would be required from (24) if the probe measured the transverse electric or magnetic field on the sphere enclosing the test antenna. In other words, the differential operator representation of the probe has enabled a reformulation of probe-corrected spherical near-field measurements that parallels and approaches the simplicity of the formulation for nearfield measurements with an ideal probe that measures the transverse electric or magnetic field. In fact, if the probe has the receiving pattern of an ideal electric dipole, it was noted after (23) that $L_{H}$ vanishes, $L_{E}$ equals a constant, and $\tilde{b}_{t}$ is proportional to $\bar{E}_{t}^{A} \times \hat{r}$; thus (29) reduces directly to the result that would be obtained from (24a) with measured $\bar{E}_{t}^{A}$. Similarly, for a probe with the receiving pattern of an ideal magnetic dipole, $\bar{b}_{t}$ is proportional to $\bar{H}_{t}^{A}$ and (29) reduces directly to the result that would be obtained from (24b) with measured $\bar{H}_{t}^{A}$.

Although they may not have the receiving pattern of a single electric or magnetic dipole, the probes most commonly used for near-field scanning are open-ended waveguide or small horns which require very few modal coefficients $C_{l}^{E}$ and $C^{H}$ to approximate well their complex receiving patterns (far fields). For example, scalar horns whose far fields can be approximated by superimposing the far fields of crossed electric and magnetic dipoles have just two nonzero modal coefficients, $C_{1}^{E}$ and $C_{1}^{H}$, and thus both $L_{E}$ and $L_{H}$ reduce to constants. Such Huygens-source probes have been used by Wood [23] for spherical scanning at intermediate distances. Equations (23) and (21) show immediately how probe correction with these scalar horns simplifies, and (27) provides a straightforward transmission formula that can be used at nearin as well as intermediate distances.

The far fields of open-ended rectangular waveguides, probably the most common and convenient near-field probes, can be well-approximated with just three modal coefficients [24] $C_{1}^{E}, C_{1}^{H}$, and $C_{2}^{H}$; thus from (21) and (22) $L_{E}$ and $L_{H}$ for openended rectangular waveguide probes reduce to

$$
\begin{gathered}
L_{E}=2 i\left(C_{1}^{E} A_{1}-3 i C_{2}^{H} A_{2}\right) \\
L_{H}=2\left(C_{1}^{H} A_{1}+6 C_{2}^{H} A_{2} \frac{1}{i k} \frac{\partial}{\partial r}\right) .
\end{gathered}
$$

It is emphasized that, even though the near fields close to as well as within the material of the probe may differ drastically from the near fields of the finite number of multipoles used to approximate the far fields of the probe, the probe responds to an arbitrary incident field (produced by sources that do not "encroach" upon the probe with respect to the radial direction) as if it were the finite number of multipoles located at the origin $0^{\prime}$ to which the far field of the probe is referenced. Moreover, physically different antennas with the same complex receiving pattern (far field) respond identically to such an arbitrary incident field. These rather remarkable conclusions follow directly from the result that the differential operator representation (16) expresses the response of an arbitrary receiving antenna in terms of the spherical mode coefficients $\left(C_{l m}^{E}, C_{m}^{H}\right)$ of the receiving pattern (far field) of the antenna.

\section{Comparison With Previous Spherical Transmission FORMULA}

In this section we show that the new spherical transmission formula (27) is consistent with the previous spherical transmission formula that was first derived by Jensen [7], and later modified by Wacker [8], [9] and Jensen [10] to allow inversion by means of generalized orthogonality relations. An excellent, comprehensive treatment and review of spherical near-field antenna measurements before 1981 may be found in the thesis by Larsen [25].

\section{A. Derivation of Jensen-Wacker Transmission Formula}

The Jensen-Wacker transmission formula may be derived from the plane-wave transmission integral. Specifically, with the probe at its reference position $(r, \phi=0, \theta=0, \psi=0)$ along the $z$-axis, and the spectrum of the test antenna written in terms of its far-field function (3c), the transmission integral (1) becomes

$$
b_{p}(r)=F i \int_{-\infty}^{\infty} \int_{-\infty}^{\infty} \bar{s}(\bar{K}) \cdot \bar{f}(\bar{K}) \frac{e^{i \gamma r}}{\gamma} d \bar{K}
$$

Similarly, when the probe is oriented at arbitrary Eulerian angles $(\phi, \theta, \psi),(32 \mathrm{a})$ may be rewritten

$b_{p}(r, \phi, \theta, \psi)=F i \int_{-\infty}^{\infty} \int_{-\infty}^{\infty} \bar{s}(\bar{K}) \cdot \bar{f}^{\prime}(\bar{K}) \frac{e^{i \gamma r}}{\gamma} d \bar{K}$

where $\bar{f}^{\prime}(\bar{K})$ is the far-field function of the test antenna rotated by the Eulerian angles $(-\psi,-\theta,-\phi)$.

The far-field function $\bar{f}$ for the test antenna may be obtained from (30a) in terms of the vector spherical harmonics,

$$
\bar{f}(\bar{K})=\frac{1}{i k} \sum_{n=1}^{\infty} \sum_{m=-n}^{n}(-i)^{n}\left[i b_{n m}^{E} \bar{N}_{n m}(\bar{K})+b_{n m}^{H} \bar{M}_{n m}(\bar{K})\right]
$$

The far-field function $\bar{f}$ ' of the "rotated" test antenna follows from (33a) and the rotational addition theorem for spherical 
waves $[26$, sec. 32$]$,

$$
\begin{aligned}
\bar{f}^{\prime}(\hat{K})=\frac{1}{i k} \sum_{n=1}^{\infty} \sum_{m, \mu=-n}^{n}(-i)^{n} \\
\cdot\left[i b_{n m}^{E} \bar{N}_{n \mu}+b_{n m}^{H} \bar{M}_{n \mu}\right] e^{i \mu \psi} d_{m n}^{n}(\theta) e^{i m \phi}
\end{aligned}
$$

where the $d_{m p}^{n}(\theta)$ are spherical rotation functions (generalizations of associated Legendre functions).

Substitution of (33b) into (32b) leads directly to the conventional form of the Jensen-Wacker transmission formula,

$$
\begin{aligned}
& b_{p}(r, \phi, \theta, \psi)=F \sum_{n=1}^{\infty} \sum_{m, \mu=-n}^{n} \\
& \cdot\left[b_{n m}^{E} R_{n \mu}^{E}(r)+b_{n m}^{H} R_{n \mu}^{H}(r)\right] e^{i \mu \psi} d_{m \mu}^{n}(\theta) e^{i m \phi} .
\end{aligned}
$$

The translated probe coefficients, which are functions only of the radial separation distance $r$ between the probe and test antennas, are defined here explicitly by the integrals

$$
\begin{aligned}
& R_{n \mu}^{E}=\frac{i(-i)^{n}}{k} \int_{-\infty}^{\infty} \int_{-\infty}^{\infty} \bar{s}(\bar{K}) \cdot \bar{N}_{n \mu}(\bar{K}) \frac{e^{i \gamma r}}{\gamma} d \bar{K} \\
& R_{n \mu}^{H}=\frac{(-i)^{n}}{k} \int_{-\infty}^{\infty} \int_{-\infty}^{\infty} \bar{s}(\bar{K}) \cdot \bar{M}_{n \mu}(\bar{K}) \frac{e^{i \gamma r}}{\gamma} d \bar{K} .
\end{aligned}
$$

Physically, each translated probe coefficient $R_{n \mu}^{E}$ or $R_{n \mu}^{H}$ may be interpreted as the response $b_{p}$ of the probe in its reference position along the $z$-axis $(r, \phi=0, \theta=0, \psi=0)$ to the incident field of the " $n \mu$ " electric or magnetic multipole (i.e., $\bar{N}_{n \mu}$ or $\bar{M}_{n \mu}$ ), respectively, radiating from the origin 0 of the test antenna with unity amplitude.

The constants $\left(b_{n m}^{E}, b_{n m}^{H}\right)$, which were defined in (24), are the unknown coefficients of the electric and magnetic multipole fields radiated by the test antenna. In order to conform to Jensen and Wacker, throughout Section IV the arbitrary factor $A_{n m}$ normalizing the multipole fields will be chosen to make $\Delta_{n m}$ defined in (28) equal to unity; specifically let $A_{n m}=$ $(-1)^{m} \sqrt{(2 n+1)(n-m) ! / 4 \pi n(n+1)(n+m) !}$.

Generalized orthogonality relations for the angular functions in (34) demand integrations over all three.Eulerian angles $\phi, \theta$ and $\psi[8]-[10]$. Thus, the modal coefficients $\left(b_{n m}^{E}, b_{n m}^{H}\right)$ of the test antenna cannot be determined, by applying orthogonality alone to (34), from measurements with an arbitrary probe unless the probe output is recorded as a function of the spin angle $\psi$ for each value of $\phi$ and $\theta$ on the scanning sphere. Wacker [8], [9] and later Jensen [10] show that such prohibitive amounts of near-field data are not required if symmetric probes are used that have all their $R_{n_{\mu}}^{E}$ and $R_{n \mu}^{H}$ zero except for $\mu= \pm 1$. These special probes allow the direct inversion of (34) (through orthogonality) from data taken with the probe in two spin orientations $\left(\psi=0^{\circ}\right.$ and $90^{\circ}$, say) at each measurement point $(\phi, \theta)$.

Specifically, for $\mu$ restricted to \pm 1 , the probe's vector output, $\bar{b}_{t}$, defined by (19) can be written from (34) as

$$
\begin{aligned}
& \bar{b}_{t}(r, \phi, \theta) \\
& =F \sum_{n=1}^{\infty} \sum_{m=-n}^{n}\left[(\hat{\theta}+i \hat{\phi}) d_{m 1}^{n}(\theta)\left(b_{n m}^{E} R_{n 1}^{E}+b_{n m}^{H} R_{n 1}^{H}\right)\right. \\
& \left.\quad+(\hat{\theta}-i \hat{\phi}) d_{m,-1}^{n}(\theta)\left(b_{n m}^{E} R_{n_{1}-1}^{E}+b_{n m}^{H} R_{n_{1}-1}^{H}\right)\right] e^{i m \phi} .
\end{aligned}
$$

Equation (36) converts to the form of the transmission formula (27) with the help of the following expressions for the $d_{m, \pm 1}^{n}(\theta)$ rotation functions in terms of the $\phi$ and $\theta$ components of the vector spherical harmonics [12]:

$$
\begin{aligned}
d_{m, \pm 1}^{n}(\theta) e^{i m \phi}=\left(i M_{n m}^{\theta} \pm M_{n m}^{\phi}\right) & \sqrt{\frac{4 \pi}{2 n+1}} \\
= & \left(i N_{n m}^{\phi} \mp N_{n m}^{\theta}\right) \sqrt{\frac{4 \pi}{2 n+1}} .
\end{aligned}
$$

The relations (37) may be obtained simply by considering an ideal $\mu= \pm 1$ probe that measures the $\phi$-component of the electric field when in the $\psi=0$ orientation. The output of this ideal probe will equal the $\phi$-component of the electric field (24a) of the test antenna. But since the response of the probe on the $z$-axis to each multipole field $\bar{N}_{n \mu}$ or $\bar{M}_{n \mu}$ defines the translated probe coefficients $R_{n \mu}^{E}$ and $R_{n \mu}^{H}$, respectively, the $\phi$ component of (24a) immediately yields the translated coefficients for this ideal probe

$$
\begin{gathered}
R_{n 1}^{E}=R_{n,-1}^{E}=\frac{-i}{2} g_{n}(k r) \sqrt{\frac{2 n+1}{4 \pi}} \\
R_{n 1}^{H}=-R_{n,-1}^{H}=\frac{1}{2} f_{n}(k r) \sqrt{\frac{2 n+1}{4 \pi}} .
\end{gathered}
$$

When (38) is introduced into (34), and (34) is equated to $E_{\phi}^{A}$ in (24a), the relations (37) result.

Substituting (37) into (36), we find

$$
\bar{b}_{t}(r, \phi, \theta)=F \sum_{n=1}^{\infty} \sum_{m=-n}^{n}\left(B_{n m}^{E}(r) \bar{M}_{n m}+B_{n m}^{H}(r) \bar{N}_{n m}\right)
$$

where $B_{n m}^{E}(r)$ and $B_{n m}^{H}(r)$ denote radial functions defined by

$$
\begin{aligned}
B_{n m}^{E}=i & \sqrt{\frac{4 \pi}{2 n+1}} \\
& \cdot\left[b_{n m}^{E}\left(R_{n,-1}^{E}+R_{n 1}^{E}\right)+b_{n m}^{H}\left(R_{n,-1}^{H}+R_{n 1}^{H}\right)\right] \\
B_{n m}^{H}= & \sqrt{\frac{4 \pi}{2 n+1}} \\
& \cdot\left[b_{n m}^{E}\left(R_{n,-1}^{E}-R_{n 1}^{E}\right)+b_{n m}^{H}\left(R_{n,-1}^{H}-R_{n 1}^{H}\right)\right] .
\end{aligned}
$$


Equation (39) confirms the result of (27). Namely, the $\mu=$ \pm 1 probe may be viewed as an ideal probe measuring the components of an effective field $\bar{b}_{t}$, such that the effective modal coefficients $B_{n m}^{E}(r)$ and $B_{n m}^{H}(r)$ can be computed simply from the orthogonality relations (28) for the vector spherical harmonics. Once the effective modal coefficients are computed, the actual modal coefficients $b_{n m}^{E}$ and $b_{n m}^{H}$ are determined from (40).

\section{B. Evaluation of the Translated Probe Coefficients}

We have shown in (39) that the conventional form of the Jensen-Wacker spherical transmission formula (34) can be recast into the same form as the new spherical transmission formula (27) derived in Section III-B. To complete the comparison of (34) with (27), we show in this section that the expressions (40) involving the translated probe coefficients $\left(R_{n \mu}^{E}, R_{n \mu}^{H}\right)$ reduce to the radial functions in (27) involving $L_{E}$ and $L_{H}$. In the conventional formulation of the probe correction for spherical scanning [7]-[10], the translated probe coefficients $\left(R_{n \mu}^{E}, R_{n \mu}^{H}\right)$ are determined from the receiving pattern of the probe through complicated translational addition theorems for the vector spherical wave functions. In order to avoid direct manipulation of these cumbersome expressions, we evaluate the translated probe coefficients from the planewave coupling integrals of the spherical harmonics in (35).

Substituting the spherical mode expansion (6) for the receiving spectrum of the probe into (35), we obtain ${ }^{2}$

$$
\begin{gathered}
R_{n \mu}^{E}=i(-i)^{n} \sum_{l=1}^{\infty}\left[C_{l,-\mu}^{E} I_{l n}^{\mu}(r)+C_{l,-\mu}^{H} J_{l n}^{\mu}(r)\right] \\
R_{n \mu}^{H}=(-i)^{n} \sum_{l=1}^{\infty}\left[-C_{l,-\mu}^{E} J_{l n}^{\mu}(r)+C_{l,-\mu}^{H} I_{l n}^{\mu}(r)\right] .
\end{gathered}
$$

The mode-mode coupling integrals in (41) are given by

$$
\begin{aligned}
& I_{l n}^{\mu}(r)=\int_{-\infty}^{\infty} \int_{-\infty}^{\infty} \bar{N}_{l,-\mu}(\bar{K}) \cdot \bar{N}_{n \mu}(\bar{K}) \frac{e^{i \gamma r}}{\gamma k} d \bar{K} \\
& J_{l n}^{\mu}(r)=\int_{-\infty}^{\infty} \int_{-\infty}^{\infty} \bar{M}_{l,-\mu}(\bar{K}) \cdot \bar{N}_{n \mu}(\bar{K}) \frac{e^{i \gamma r}}{\gamma k} d \bar{K} .
\end{aligned}
$$

The single summation over $l$ in (4la) and (41b) reflects the vanishing of the integrals corresponding to (42) with $m \neq-\mu$. Note from (42) that $I_{l n}^{\mu}$ and $J_{l n}^{\mu}$ obey the symmetries,

$$
\begin{gathered}
I_{l n}^{\mu}=I_{n l}^{\mu}=I_{l n}^{-\mu} \\
J_{l n}^{\mu}=J_{n l}^{\mu}=-J_{l n}^{-\mu} .
\end{gathered}
$$

The mode-mode coupling integrals in (42) are evaluated in the Appendix by two different methods that lead to two different

\footnotetext{
${ }^{2}$ Equation (41) shows that each $R_{n_{\mu}}^{E}$ and $R_{n \mu}^{H}$ depends upon all the spherical multipole coefficients $\left(C_{l,-\mu}^{E}, C_{t,-\mu}^{H}\right)$ of the receiving pattern of the probe. However, if the receiving pattern of the probe can be approximated by just a few multipoles, all but a few $C$ are negligible and the summation in (41) extends over only a few values of $l$.
}

closed forms for $I_{l n}^{\mu}$ and $J_{l n}^{\mu}$. The first method leads to the closed form (53), which when substituted into (41), results in expressions for $R_{n_{\mu}}^{E}$ and $R_{n \mu}^{H}$ that agree with those obtained from the translational addition theorems for the spherical wave functions [7]-[10]. The second method of evaluation in the Appendix leads to a closed form (55) that can be used to obtain the simplified spherical transmission formula (27).

To show this, we first restrict our attention as we did in Section III-B, to the special $\mu= \pm 1$ probes with spherical mode coefficients displaying the symmetries given by (20). From (20), (41) and (43), the translated probe coefficients obey the corresponding symmetries,

$$
\begin{gathered}
R_{n \mu}^{E, H}=0 \quad \mu \neq \pm 1 \\
R_{n 1}^{E}=R_{n,-1}^{E} \equiv R_{n}^{E} \\
R_{n 1}^{H}=-R_{n,-1}^{H} \equiv R_{n}^{H}
\end{gathered}
$$

which, in turn, allow (39) and (41) to be written

$$
\begin{gathered}
\bar{b}_{t}(r, \phi, \theta)=2 F \sum_{n=1}^{\infty} \sum_{m=-n}^{n} \sqrt{\frac{4 \pi}{2 n+1}} \\
\cdot\left(i b_{n m}^{E} R_{n}^{E} \bar{M}_{n m}-b_{n m}^{H} R_{n}^{H} \bar{N}_{n m}\right) \\
R_{n}^{E}=i(-i)^{n} \sum_{l=1}^{\infty}\left(C_{l}^{E} I_{l n}^{1}-C_{l}^{H} J_{l n}^{1}\right) \\
R_{n}^{H}=-(-i)^{n} \sum_{l=1}^{\infty}\left(C_{l}^{E} J_{l n}^{1}+C_{l}^{H} I_{l n}^{1}\right) .
\end{gathered}
$$

Finally, substitution of (55) from the Appendix into (45) reveals the spherical transmission formula (27), and thus completes the demonstration that the spherical transmission formula (27) is equivalent to the previous transmission formula (34) based on the rotational and translational addition theorems for spherical waves.

\section{APPENDIX}

\section{Evaluation of Mode-Mode Coupling Integrals}

We wish to evaluate the integrals in (42) of the main text:

$$
\begin{gathered}
I_{l n}^{\mu}(r)=\int_{-\infty}^{\infty} \int_{-\infty}^{\infty} \bar{N}_{l,-\mu}(\bar{K}) \cdot \bar{N}_{n \mu}(\bar{K}) \frac{e^{i \gamma r}}{\gamma k} d \bar{K} \\
J_{l n}^{\dot{\mu}}(r)=\int_{-\infty}^{\infty} \int_{-\infty}^{\infty} \bar{M}_{l,-\mu}(\bar{K}) \cdot \bar{N}_{n \mu}(\bar{K}) \frac{e^{i \gamma r}}{\gamma k} d \bar{K} .
\end{gathered}
$$

With a change of variables from $k_{x}$ and $k_{y}$ to $\eta=\gamma / k$ and $\phi$ $=\tan ^{-1}\left(k_{y} / k_{x}\right)$,

$$
\int_{-\infty}^{\infty} \int_{-\infty}^{\infty} d k_{x} d k_{y} \rightarrow k^{2} \int_{0}^{2 \pi} d \phi \int_{i \infty}^{1} \eta d \eta
$$

where the contour in the $\eta$-plane may be taken as the imaginary axis between $\eta=i \infty$ and $\eta=0$, and the real axis 
between $\eta=0$ and $\eta=1$. Equations (46) may be written as

$$
\begin{gathered}
I_{l n}^{\mu}=2 \pi \int_{i \infty}^{1} \bar{N}_{l,-\mu} \cdot \bar{N}_{n \mu} e^{i \rho \eta} d \eta \quad(\rho=k r) \\
J_{l n}^{\mu}=2 \pi \int_{i \infty}^{1} \bar{M}_{l,-\mu} \cdot \bar{N}_{n \mu} e^{i \rho \eta} d \eta
\end{gathered}
$$

because the integrands in (46) are azimuthally symmetric, and thus the $\phi$-integrals merely produce a factor of $2 \pi$. Substitution into (48) of the components of $\bar{M}$ and $\bar{N}$ from (7) of the main text yields, after a straightforward manipulation that uses the differential equation for $P_{n}^{\mu}$,

$$
\begin{aligned}
& I_{l n}^{\mu}= \pi(-1)^{\mu} A_{l \mu} A_{n \mu} \\
& \cdot \int_{i \infty}^{1}\{[l(l+1)+n(n+1) \\
&\left.\left.\left.+\frac{d}{d \eta}(1-\eta)^{2}\right) \frac{d}{d \eta}\right]\left(P_{l}^{\mu} P_{n}^{\mu}\right)\right\} e^{i \rho \eta} d \eta \\
& J_{l n}^{\mu}=2 \pi \mu i(-1)^{\mu} A_{l \mu} A_{n \mu} \int_{i \infty}^{1}\left[\frac{d}{d \eta}\left(P_{l}^{\mu} P_{n}^{\mu}\right)\right] e^{i \rho \eta} d \eta .
\end{aligned}
$$

Partial integrations with respect to $\eta$ and differentiations with respect to $\rho$ transform (49) into

$$
\begin{aligned}
I_{l n}^{\mu}= & \pi(-1)^{\mu} A_{l \mu} A_{n \mu} \\
& \cdot\left[l(l+1)+n(n+1)-\rho^{2}\left(1+\frac{2}{\rho} \frac{d}{d \rho}+\frac{d^{2}}{d \rho^{2}}\right)\right] \\
& \cdot \int_{i \infty}^{1} P_{l}^{\mu} P_{n}^{\mu} e^{i \rho \eta} d \eta \\
& J_{l n}^{\mu}=2 \pi \mu(-1)^{\mu} A_{l \mu} A_{n \mu} \rho \int_{i \infty}^{1} P_{l}^{\mu} P_{n}^{\mu} e^{i \rho \eta} d \eta .
\end{aligned}
$$

It follows from Rodrigues' formula ((8a) of main text) that $P_{f}^{\mu} P_{n}^{\mu}$ is a polynomial in $\eta$ and hence may be expanded in terms of Legendre polynomials $P_{\nu}(\eta)$. This expansion may be written in the form [26, eqs. (34.39) and (25.19)]

$$
A_{l \mu} A_{n \mu} \sqrt{\Delta_{l \mu} \Delta_{n \mu}} P_{l}^{\mu} P_{n}^{\mu}=\sum_{\nu=|l-n|}^{1+n}\langle\ln ; \mu \mid \nu\rangle P_{\nu}
$$

$\langle l n ; \mu \mid \nu\rangle=$

$$
\frac{(-1)^{\mu}}{4 \pi} \sqrt{\frac{(2 l+1)(2 n+1)}{l(l+1) n(n+1)}}(2 \nu+1)\left(\begin{array}{c}
\ln \nu \\
-\mu \mu 0
\end{array}\right)\left(\begin{array}{c}
\ln \nu \\
000
\end{array}\right)
$$

where the $\Delta$ are defined in (28) of the main text. (For the normalization chosen in Section IV the $\Delta$ equal unity.) The 3

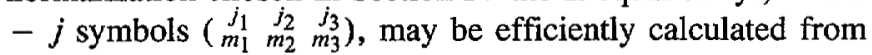
recursion formulas, although useful closed-form expressions for $\langle l n ; \mu \mid \nu\rangle$ exist for the special cases $\mu=0$ and 1 [27]. Note that $\langle l n ; \mu \mid \nu\rangle$ is zero if $\nu<|l-n|$ or $\nu>l+n$, or if $l+n$ $+\nu$ is odd.
It is now apparent from the integral representation of the spherical Hankel function

$$
h_{n}^{(1)}(\rho)=(-i)^{n} \int_{i \infty}^{1} P_{n}(\eta) e^{i \rho \eta} d \eta
$$

and the expansion (51) that the integrals in (50) may be performed analytically to obtain

$$
\begin{aligned}
I_{l n}^{\mu}= & \frac{\pi(-1)^{\mu}}{\sqrt{\Delta_{l \mu} \Delta_{n \mu}}} \sum_{\nu} \\
& \cdot i^{\nu}[l(l+1)+n(n+1)-\nu(\nu+1)]\langle l n ; \mu \mid \nu\rangle h_{\nu}^{(1)}(\rho) \\
& J_{l n}^{\mu}=\frac{2 \mu \rho \pi(-1)^{\mu}}{\sqrt{\Delta_{l \mu} \Delta_{n \mu}}} \sum_{\nu} i^{\nu}\langle l n ; \mu \mid \nu\rangle h_{\nu}^{(1)}(\rho)
\end{aligned}
$$

where use has been made of the differential equation satisfied by the Hankel function.

When $\mu$ is equal to 1 , the formulas

$$
\begin{gathered}
\frac{1}{2}\left\{l(l+1)+n(n+1)+\frac{d}{d \eta}\left(1-\eta^{2}\right) \frac{d}{d \eta}\right\} P_{l}^{1} P_{n}^{1} \\
=n(n+1)\left[\left(\eta \frac{d^{2} P_{l}}{d \eta^{2}}+\frac{d P_{l}}{d \eta}\right)\right. \\
\left.\cdot\left(n \eta P_{n}+P_{n-1}\right) /(n+1)-\frac{d^{2} P_{l}}{d \eta^{2}} P_{n}\right]
\end{gathered}
$$

and

$$
\begin{aligned}
& \frac{d}{d \eta}\left(P_{l}^{1} P_{n}^{1}\right)=n(n+1)\left[\frac{d^{2} P_{l}}{d \eta^{2}}\right. \\
& \left.\cdot\left(n \eta P_{n}+P_{n-1}\right) /(n+1)-\left(\eta \frac{d^{2} P_{l}}{d \eta^{2}}+\frac{d P_{l}}{d \eta}\right) P_{n}\right]
\end{aligned}
$$

convert (49) to

$$
\begin{aligned}
& I_{l n}^{1}=\frac{i^{n}}{2 l(l+1)} \sqrt{\frac{(2 l+1)(2 n+1)}{\Delta_{l 1} \Delta_{n 1}}}\left[i F_{l} g_{n}+G_{l} f_{n}\right] \\
& J_{l n}^{1}=\frac{i^{n}}{2 l(l+1)} \sqrt{\frac{(2 l+1)(2 n+1)}{\Delta_{l 1} \Delta_{n 1}}}\left[i F_{l} f_{n}-G_{l} g_{n}\right]
\end{aligned}
$$

where $\left(F_{l}, G_{l}\right)$ and $\left(f_{n}, g_{n}\right)$ are defined in (22) and (25) of the main text. Equations (55) are derived by moving the factors of (54) dependent on $l$ outside the integrals of (49) as differential operators. Integration of the remaining $n$-dependent factors is accomplished using (52).

\section{REFERENCES}

[1] M. K. Hu, "Near-zone transmission formulas," IRE Nat. Conv. Record, vol. 6, pt. 8, pp. 128-138, 1958.

[2] W. L. Weeks, Electromagnetic Theory for Engineering Applications. New York: Wiley, 1964, p. 332.

[3] D. M. Kerns, "General formula for voltage induced in a receiving antenna," in Digest Nat. Radio Sci. Meeting, Boulder, CO, Nov. 1978.

[4] , "Plane-wave scattering-matrix theory of antennas and antenna- 
antenna interactions," Nat. Bur. Stand. Monograph 162, June 1981.

[5] A. D. Yaghjian, "Efficient computation of antenna coupling and fields within the near-field region," IEEE Trans. Antennas Propagat., vol. AP-30, pp. 113-128, Jan. 1982.

[6] J. A. Stratton, Electromagnetic Theory. New York: McGraw Hill, 1941, ch. 7.

[7] F. Jensen, "Electromagnetic near-field far-field correlations," Ph.D. dissertation, Tech. Univ. Denmark, Lyngby, 1970.

[8] P. F. Wacker, "Near-field antenna measurements using a spherical scan: Efficient data reduction with probe correction," in Inst. Elec. Eng. Conf. Pub. 113, Conf. Precision Electromagn. Measurements, London, July 1974, pp. 286-288.

[9] …, "Non-planar near-field measurements: Spherical scanning,' Nat. Bur. Stand. Rep. NBSIR 75-809, June 1975.

[10] F. Jensen, "On the probe compensation for near-field measurements on a sphere," $A E U$, vol. 29, pp. 305-308, July/Aug-1975.

[11] A. D. Yaghjian, "Simplified approach to probe-corrected spherical near-field scanning," Electron. Lett., vol. 20, pp. 195-196, 1 March 1984. (Also in Digest Int. Symp. Antennas Propagat., Boston, MA, June 1984, pp. 670-673.)

[12] R. C. Wittmann, "Probe correction in spherical near-field scanning, viewed as an ideal probe measuring an effective field," in Digest Int. Symp. Antennas Propagat., Boston, MA, June 1984, pp. 674-677.

[13] J. D. Jackson, Classical Electrodynamics. New York: Wiley, 1962 , 1975 , ch. 16.

[14] J. Appel-Hansen, "Antenna measurements," in The Handbook of Antenna Design, vol. 1. London: Peregrinus, 1982, ch. 8.

[15] D. M. Kerns, "Correction of near-field antenna measurements made with an arbitrary but known measuring antenna," Electron. Lett., vol. 6, pp. 346-347, May 1970.

[16] C. F. Stubenrauch and A. C. Newell, "Some recent near-field antenna measurements at NBS," Microwave J., vol. 23, pp. 37-42, Nov. 1980.

[17] A. C. Newell and A. Repjar, "Results of spherical near-field measurements on narrow beam antennas," in Digest Int. Symp. Antennas Propagat., Stanford, CA, June 1977, pp. 382-385.

[18] D. T. Paris, W. M. Leach, and E. B. Joy, "Basic theory of probecompensated near-field measurements," IEEE Trans. Antennas Propagat., vol. AP-26, pp. 373-379, May 1978.

[19] W. M. Leach, Jr. and D. T. Paris, "Probe compensated near-field measurements on a cylinder," IEEE Trans. Antennas Propagat., vol. AP-2, pp. 435-445, July 1973.

[20] A. D. Yaghjian, "Near-field antenna measurements on a cylindrical surface: A source scattering-matrix formulation," Nat. Bur. Stand. Tech. Note 696, Sept. 1977.

[21] G. V. Borgiotti, "Integral equation formulation for probe corrected far-field reconstruction from measurements on a cylinder," IEEE Trans. Antennas Propagat., vol. AP-26, pp. 572-578, July 1978.

[22] F. H. Larsen, "Probe correction of spherical near-field measurements," Electron. Lett., vol. 13, pp. 393-395, July 1977.

[23] P. J. Wood, "'The prediction of antenna characteristics from spherical near-field measurements-Parts I and II, theory and experimental verification," Marconi Rev., vol. 30, pp. 117-155, 1977.

[24] A. D. Yaghjian, "Approximate formulas for the far field and gain of open-ended rectangular waveguide," IEEE Trans. Antennas Propagat., vol. AP-32, pp. 378-384, Apr. 1984.

[25] F. H. Larsen, "Probe-Corrected Spherical Near-Field Antenna Measurements," Tech. Univ. Denmark Rep. LD 36, Dec. 1980.

[26] K. Gottfried, Quantum Mechanics, Vol. 1: Fundamentals. New York: Benjamin, 1966.

[27] J. H. Bruning and Y. T. Lo, "Multiple scattering of EM waves by spheres, Part I-multipole expansion and ray-optical solutions," IEEE Trans. Antennas Propagat., vol. AP-19, pp. 378-390, May 1971.

Arthur D. Yaghjian (S'68-M'69-SM'84), for a photograph and biography please see page 5 of the January 1984 issue of this TRANSACTIONS.

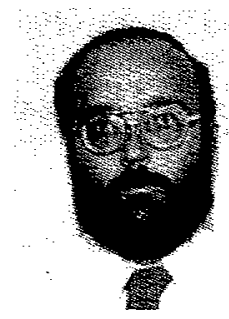

Ronald C. Wittmann was born in Seattle, WA, on January 9,1950 . He received the B.S. degree in physics from the University of Washington, Seattle, in 1972, and the M.S. degree in physics from the University of Colorado, Boulder, in 1976.

Since 1978 he has been employed in the Electromagnetic Fields Division of the National Bureau of Standards, Boulder, CO. His major research has been in the areas of remote sensing and near-field antenna measurements. 